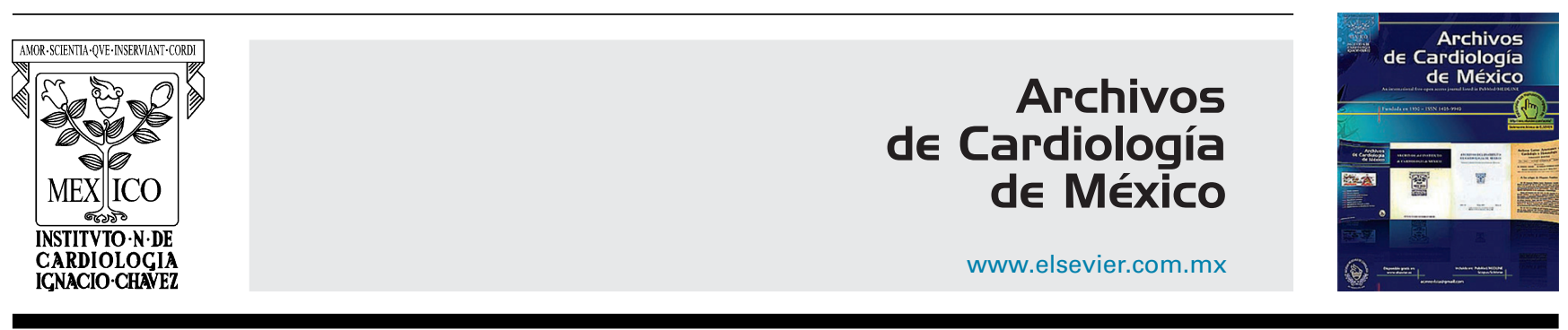

INVESTIGACIÓN CLÍNICA

\title{
Nivel de ruido ambiental en 2 unidades de cuidados críticos de un centro de tercer nivel de atención
}

\author{
José Manuel Ornelas-Aguirre ${ }^{a, b, *}$, Olivia Zárate-Coronado ${ }^{c}$, \\ Fabiola Gaxiola-González ${ }^{d}$ y Venigna Neyoy-Sombra ${ }^{d}$
}

\begin{abstract}
a División de Investigación en Salud, Hospital de Especialidades No. 2, Unidad Médica de Alta Especialidad, Centro Médico Nacional del Noroeste, Instituto Mexicano del Seguro Social, ciudad Obregón, Sonora, México

b Departamento de Ciencias de la Salud, Universidad de Sonora, Campus Cajéme, Obregón, Sonora, México

' Dirección de enfermería, Hospital de Especialidades No. 2, Unidad Médica de Alta Especialidad, Centro Médico Nacional del Noroeste, Instituto Mexicano del Seguro Social, Obregón, Sonora, México

d Servicio de urgencias y terapia intensiva, Hospital de Especialidades No. 2, Unidad Médica de Alta Especialidad, Centro Médico Nacional del Noroeste, Instituto Mexicano del Seguro Social, Obregón, Sonora, México
\end{abstract}

Recibido el 25 de octubre de 2016; aceptado el 27 de febrero de 2017

\section{PALABRAS CLAVE \\ Ruido; \\ Unidad de cuidado \\ intensivo; \\ Calidad de atención \\ en salud; \\ México}

\begin{abstract}
Resumen
Introducción: La Organización Mundial de la Salud establece como nivel máximo de ruido 40 decibelios $(\mathrm{dB})$ para una unidad de cuidados críticos. El objetivo de este estudio fue comparar la intensidad del nivel de ruido ambiental en 2 unidades de cuidados críticos de un hospital de tercer nivel de atención.

Métodos: En un diseño observacional, descriptivo y transversal se comparó el nivel máximo de ruido dentro de la unidad de cuidados intensivos metabólicos y coronarios con un medidor digital. La medición en 4 puntos diferentes de cada habitación con intervalos de 5 min durante un período de 60 min se llevó a cabo a las 7:30, 14:30 y 20:30. El promedio de las observaciones se comparó con estadística descriptiva y U de Mann-Whitney. Un análisis con la prueba de Kruskal-Wallis se realizó para comparar los promedios de ruido en las diferentes lecturas.

Resultados: Se observó que el ruido en la unidad de cuidados intensivos tuvo un promedio de $64.77 \pm 3.33 \mathrm{~dB}(\mathrm{p}=0.08)$; algo similar sucedió en la unidad de cuidados intensivos metabólicos coronarios con un promedio de $60.20 \pm 1.58 \mathrm{~dB}(\mathrm{p}=0.129)$. El $25 \%$ o más de las mediciones superaron hasta por 20 puntos lo recomendado por la Organización Mundial de la Salud.
\end{abstract}

\footnotetext{
* Autor para correspondencia. Universidad de Sonora, campus Cajéme. Blvd. Bordo Nuevo S/N. Antiguo Ejido Providencia. Ciudad Obregón, Sonora. México 85010 Teléfono: +52 (64) 4462-0471; Fax: +52 6444134590.

Correo electrónico: jose.ornelas@cajeme.uson.mx (J.M. Ornelas-Aguirre).
} 


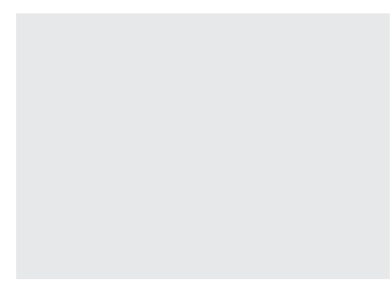

\section{KEYWORDS}

Noise; Intensive care unit; Quality of health care; Mexico.
Conclusiones: El nivel de ruido que se encontró en las unidades de cuidados intensivos estudiadas sobrepasan el nivel máximo recomendado para un hospital. Es necesario diseñar y aplicar acciones para una mayor participación del personal de salud en la disminución del ruido ambiental.

(C) 2017 Instituto Nacional de Cardiología Ignacio Chávez. Publicado por Masson Doyma México S.A. Este es un artículo Open Access bajo la licencia CC BY-NC-ND (http://creativecommons. org/licenses/by-nc-nd/4.0/).

\section{Environmental noise levels in 2 intensive care units in a tertiary care centre}

\begin{abstract}
Introduction: The World Health Organisation (WHO) has established a maximum noise level of 40 decibels $(\mathrm{dB})$ for an intensive care unit. The aim of this study was to compare the noise levels in 2 different intensive care units at a tertiary care centre.

Methods: Using a cross-sectional design study, an analysis was made of the maximum noise level was within the intensive coronary care unit and intensive care unit using a digital meter. A measurement was made in 4 different points of each room, with 5 minute intervals, for a period of 60 minutes $7: 30,14: 30$, and 20:30. The means of the observations were compared with descriptive statistics and Mann-Whitney U. An analysis with Kruskal-Wallis test was performed to the mean noise level.

Results: The noise observed in the intensive care unit had a mean of $64.77 \pm 3.33 \mathrm{~dB}(P=.08)$, which was similar to that in the intensive coronary care unit, with a mean of $60.20 \pm 1.58 \mathrm{~dB}$ $(P=.129)$. Around $25 \%$ or more of the measurements exceeded the level recommended by the WHO by up to 20 points.

Conclusions: Noise levels measured in intensive care wards exceed the maximum recommended level for a hospital. It is necessary to design and implement actions for greater participation of health personnel in the reduction of environmental noise.

(C) 2017 Instituto Nacional de Cardiología Ignacio Chávez. Published by Masson Doyma México S.A. This is an open access article under the CC BY-NC-ND license (http://creativecommons. org/licenses/by-nc-nd/4.0/).
\end{abstract}

\section{Introducción}

Las unidades de cuidados críticos (UCC) se caracterizan por su eficacia técnica, el uso de tecnología de punta y una atención con un bajo grado de relaciones personales ${ }^{1}$. El incremento de equipos automatizados que contaminan con ruido, vibración y alarmas sonoras pueden alterar a los enfermos, dejando una mala experiencia de la atención recibida ${ }^{2}$. Las guías de la Organización Mundial de la Salud recomiendan que los niveles de ruido no superen los 40 decibelios $(\mathrm{dB})$ dentro de los hospitales para asegurar un adecuado descanso de los enfermos ${ }^{3}$.

En una encuesta realizada a familiares y enfermos en 4 hospitales de España se encontró que el 13\% de los participantes consideraban el ruido como insoportable dentro del hospital ${ }^{4}$. En consecuencia, un ambiente que debiera de ser tranquilo, se ha convertido en ruidoso y estresante para enfermos y familiares ${ }^{5}$. Así lo reveló un estudio realizado por Freedman et al., quienes reportaron que los enfermos encontraron las intervenciones de enfermería (aspiraciones, punciones, toma de muestras, etc.) y el ruido como los principales factores estresantes durante su estancia en la UCC6 ${ }^{6}$.

La evidencia de los efectos del ruido ambiental en la salud se asocia a alteraciones del sueño y rendimiento cognitivo en adultos y niños ${ }^{7}$. La exposición ininterrumpida al ruido continuo en niveles cercanos a $60 \mathrm{~dB}$ provoca la activación fisiológica, incluyendo aumento de la frecuencia cardíaca, la presión arterial ${ }^{8}$, confusión, irritabilidad, disminución de la audición, así como baja capacidad de concentración $^{9,10}$; más aun, algunos estudios han mostrado alteración del cortisol, el colesterol total, los triglicéridos ${ }^{11}$, la viscosidad de la sangre, el recuento de plaquetas y los niveles de glucosa en la sangre ${ }^{12}$ en relación con el ruido ambiental.

Un estudio realizado por Lawson et al. en 2010 reportó que hasta un $57 \%$ de los sobresaltos y despertares en enfermos que duermen dentro de las unidades de cuidados críticos se deben al ruido ${ }^{13}$. Este hecho ya se había reportado por Aaron et al. desde 1996, al mencionar que los elevados niveles de ruido en las unidades de cuidados críticos junto con los síntomas de la enfermedad impactan en la capacidad para descansar y dormir en el enfermo ${ }^{14}$. Si a esto le agregamos que el ambiente propio de la UCC afecta psicológicamente a los pacientes de un modo negativo ${ }^{15}$, se incrementa el riesgo de complicaciones y estrés en el enfermo. A este respecto y centrado en la calidad del sueño en los pacientes de una UCC, Nicolás et al. en 2008 realizaron un estudio en el que se plantearon como objetivo describir cómo perciben los pacientes su sueño nocturno en la UCC ${ }^{16}$, utilizando para ello el cuestionario de sueño de Richards-Campbell ${ }^{17}$. Los resultados mostraron que el $28 \%$ de los pacientes valoraron su sueño como bueno, el $46 \%$ regular y el $26 \%$ como malo. Los autores concluyeron que el perfil de sueño de los pacientes de la UCC analizado se caracterizó por ser ligero, 
con despertares frecuentes e insuficiente en la mayoría de los $\operatorname{casos}^{17}$.

Dentro de los motivos de ruido en un hospital se encuentran los siguientes: sistemas de aire acondicionado, lámparas fluorescentes de techo, alarmas sonoras en equipos y monitores ${ }^{18}$, apertura y cierre de puertas, sonido de teléfonos, caída de agua en fregaderos, trabajos de limpieza y paso de carritos que transportan ropa y comida ${ }^{13}$.

El ruido se ha convertido en una amenaza a la que se le ha dado poca importancia, y se asocia a un mayor riesgo de estrés e insomnio en enfermos crónicos y ancianos ${ }^{14,19}$. Además de ser un riesgo constante para la buena relación médico-paciente y el desarrollo de actividades de educación e investigación ${ }^{20}$. Este hecho ha originado la realización de diversos estudios en diferentes regiones del mundo ${ }^{20-26}$ (tabla 1).

En México e Iberoamérica, no han sido suficientemente estudiadas las implicaciones que el ruido tiene sobre la atención de los enfermos. Se considera que es importante el estudio del ruido dentro de los hospitales, por las implicaciones que tiene en la calidad de la atención y el cumplimiento de los estándares de certificación sanitaria ${ }^{27}$; por lo tanto, el objetivo de este estudio fue comparar el nivel de ruido ambiental en 2 UCC de un hospital de tercer nivel de atención.

\section{Métodos}

En un diseño transversal analítico, descriptivo y comparativo realizado del 1 de enero al 30 de marzo de 2013, se analizó el nivel de ruido ambiental en 2 UCC en un centro médico de tercer nivel de atención. Se solicitó autorización al comité de ética e investigación (R-2012-2602-46) y al departamento de Seguridad e Higiene del hospital antes de que el estudio se iniciara. A los investigadores participantes se les proporcionó información común y estandarizada en relación con la información a obtener y las pruebas a realizar.

\section{Medición del nivel de ruido}

Para tomar las lecturas del ruido ambiental se utilizó un medidor digital fabricado por Extech Instruments Corporation $^{\circledR}$ modelo-407730 con una precisión $\pm 2 \mathrm{~dB}$ con resolución de $0.1 \mathrm{~dB}$ y un rango de lectura de $40 \sim 130 \mathrm{~dB}$. La medición del nivel de ruido se evaluó entre semana y durante el fin de semana. Antes de realizar las mediciones, el equipo fue calibrado de acuerdo a las indicaciones del fabricante. Se evitó comentar al personal de los servicios involucrados lo que se pretendía realizar para evitar que modificaran su comportamiento durante la evaluación (efecto Hawthorne). Cada una de las mediciones se realizó en horarios diferentes (7:30, $14: 30$ y $20: 30)$ y coincidían con el enlace de turno y visita de familiares a pacientes hospitalizados, siendo esos horarios los de mayor tránsito de personas. Al final, se calculó el promedio de las mediciones obtenidas de lunes a viernes y durante el fin de semana, así como los valores máximos y mínimos para el ruido ambiental. Todas las mediciones se realizaron durante $60 \mathrm{~min}$ continuos con lecturas cada 5 min en al menos 4 puntos diferentes de cada una de las UCC del hospital (una unidad de cuidados intensivos metabólicos [UCI] y otra de cuidados coronarios [UCIC]).

\section{Declaración ética}

Este estudio fue aprobado por el Comité Local de Investigación en Salud número 2602 del Hospital de Especialidades No. 2, Unidad Médica de Alta Especialidad del Instituto Mexicano del Seguro Social en el Centro Médico Nacional del Noroeste de ciudad Obregón, Sonora. México. Los autores declaran haber seguido los protocolos establecidos por el Instituto Mexicano del Seguro Social para acceder a los datos de las personas incluidas en este estudio y que la información obtenida fue utilizada solo con fines de divulgación científica. Todos los autores leyeron y aprobaron el manuscrito.

\section{Análisis estadístico}

La información fue capturada en una base de datos previamente diseñada para este propósito y el análisis se realizó utilizando el paquete estadístico SPSS para Windows ${ }^{\circledR}$ versión 19.0. Para las variables numéricas y de razón se calcularon frecuencias absolutas y relativas, promedio \pm desviación estándar, valores máximos y mínimos, así como percentiles 5, 10, 25, 50 y 75 para los niveles de ruido. Para la comparación intragrupo de los promedios de ruido por horario y unidad de cuidado crítico se utilizó la prueba de $U$ de Mann-Whitney en virtud de que el análisis inicial con la prueba de Kolmogorov-Smirnov demostró que no existía normalidad en la población de estudio $(p=0.0001)$. Se realizó un análisis con la prueba de Kruskal-Wallis a las diferentes mediciones obtenidas, donde un valor de $p \leq 0.05$ fue considerado como estadísticamente significativo.

\section{Resultados}

Se encontró un promedio de ruido entre semana de $63.40 \pm 2.82 \mathrm{~dB}$, con un valor mínimo de $60.30 \mathrm{~dB}$ y máximo de $65.37 \mathrm{~dB}$, muy superior a lo recomendado por la Organización Mundial de la Salud. Es de hacer notar que durante el fin de semana, donde se esperaba un descenso significativo del ruido, esto no sucedió.

La UCl mantuvo los niveles de ruido más elevados con un promedio entre semana de $64.77 \pm 3.33 \mathrm{~dB}$ y el fin de semana de $62.83 \pm 1.62 \mathrm{~dB}(\mathrm{p}=0.513)$; en segundo lugar la UCIC con niveles de ruido entre semana de $60.20 \pm 1.58 \mathrm{~dB}$ y $59.25 \pm 1.99 \mathrm{~dB}(\mathrm{p}=0.275)$ el fin de semana (tabla 2$)$.

Se observó que para la UCIC durante la semana solo el $75 \%$ de las mediciones superaban esta cifra (P25 =59.72, $\mathrm{P} 50=61.07, \mathrm{P} 75=61.12$ ) y durante el fin de semana el $50 \%$ de las mediciones lo superaba $(P 50=59.83$, P75 $=60.36)$. En cambio, en la UCl entre semana y durante el fin de semana siempre fue superado este valor (tabla 3 ).

Por último, se observa una disminución en el nivel de ruido durante el fin de semana en comparación con el trabajo entre semana (fig. 1).

\section{Discusión}

Los resultados de este estudio son consistentes con niveles de ruido dentro de las UCC que superan en más de un $40 \%$ lo recomendado por la Organización Mundial de la Salud ${ }^{3}$ 
(A)

75

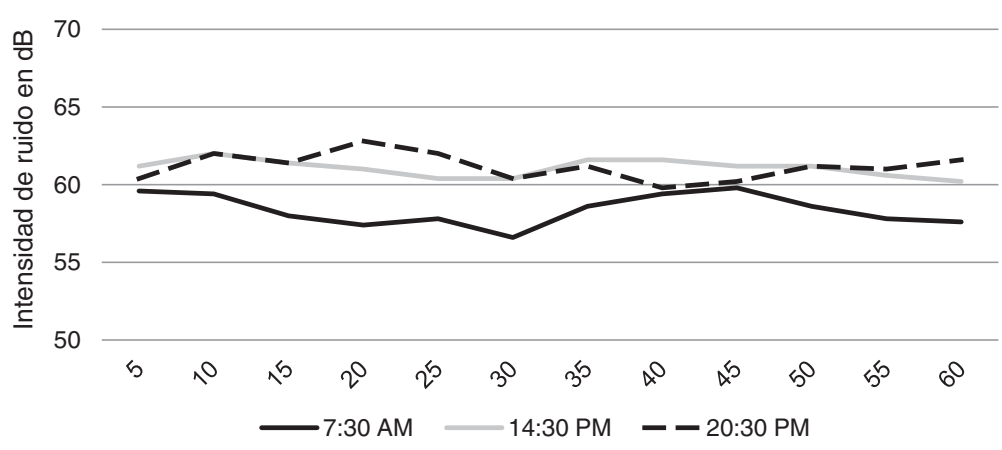

(C)

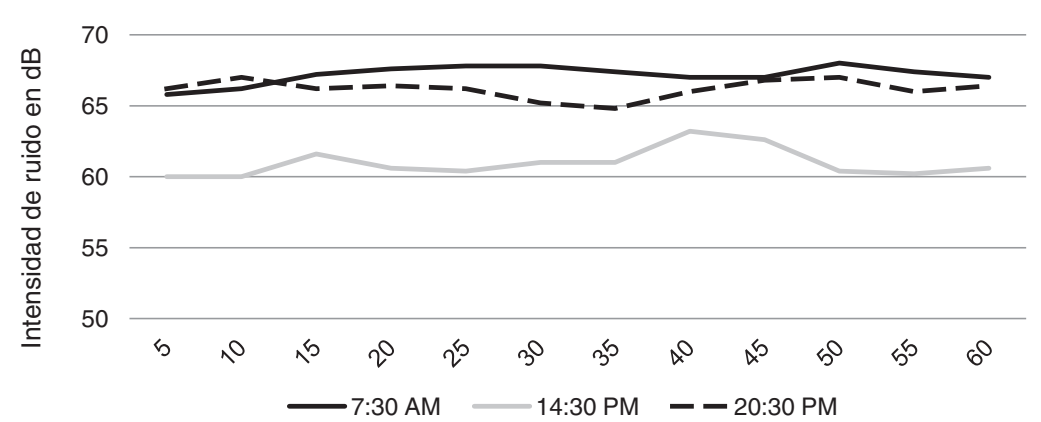

(B)

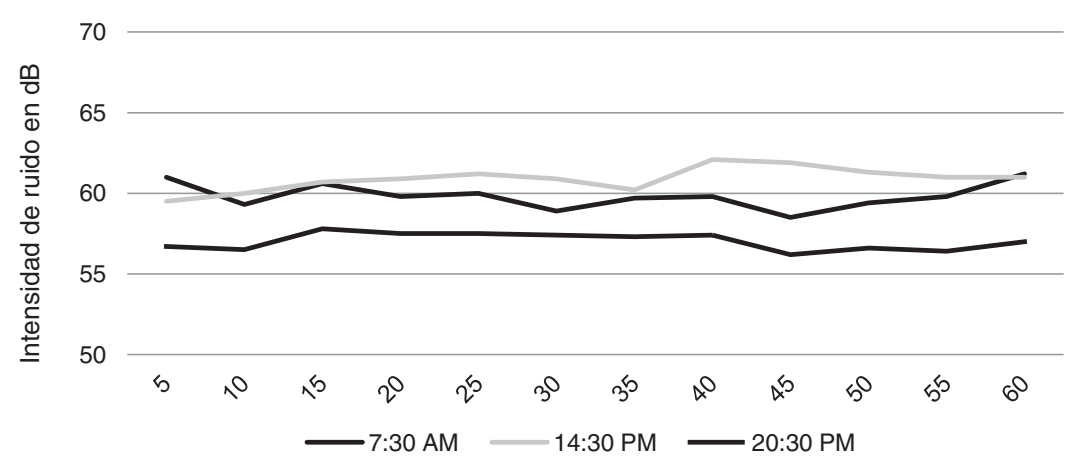

(D)

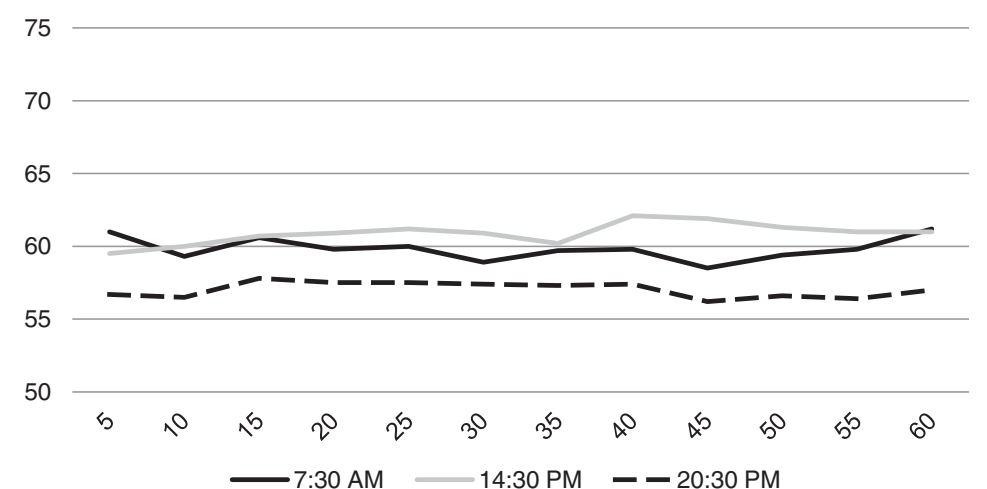

Figura 1 Niveles de ruido en decibelios (dB) por turno laboral en las unidades de cuidados intensivos metabólicos y coronarios.

$1 \mathrm{~A}$. Intensidad de ruido (dB) en UCIC (entre semana). Todas las lecturas se tomaron cada 5 min durante $1 \mathrm{~h}$ en 3 ocasiones diferentes durante los horarios definidos; se presentan los promedios de cada lectura.

1B. Intensidad de ruido (dB) en UCIC (fin de semana). Todas las lecturas se tomaron cada 5 min durante $1 \mathrm{~h}$ en 3 ocasiones diferentes durante los horarios definidos; se presentan los promedios de cada lectura.

$1 \mathrm{C}$. Intensidad de ruido (dB) en UCI (entre semana). Todas las lecturas se tomaron cada 5 min durante $1 \mathrm{~h}$ en 3 ocasiones diferentes durante los horarios definidos; se presentan los promedios de cada lectura.

1D. Intensidad de ruido (dB) en UCI (fin de semana). Todas las lecturas se tomaron cada 5 min durante $1 \mathrm{~h}$ en 3 ocasiones diferentes durante los horarios definidos; se presentan los promedios de cada lectura.

UCI: unidad de cuidados intensivos; UCIC: unidad de cuidados intensivo coronarios. 
Tabla 1 Comparación de algunos estudios previos acerca del nivel de ruido en hospitales

\begin{tabular}{|c|c|c|c|c|c|}
\hline Autores & Año & Lugar & $\begin{array}{l}\text { Camas en el } \\
\text { hospital }\end{array}$ & $\begin{array}{l}\text { Nivel de ruido } \\
\text { encontrado } \\
\text { (dB) }\end{array}$ & $\begin{array}{l}\text { Servicios } \\
\text { analizados }\end{array}$ \\
\hline Vinodhkumaradithyaa et $\mathrm{al}^{20}$ & 2008 & India & 2,200 & $\begin{array}{l}64-70 \\
65-80\end{array}$ & $\begin{array}{l}\text { Hospitalización } \\
\text { Salas de cirugía }\end{array}$ \\
\hline Vílchez-Dagostino et $\mathrm{al}^{21}$ & 2012 & Perú & 439 & $58-70$ & Hospitalización \\
\hline Yoder et $\mathrm{al}^{22}$ & 2012 & EE. UU. & 550 & 70 & Hospitalización \\
\hline McLaren y Maxwell-Armstrong ${ }^{23}$ & 2008 & Inglaterra & 1,700 & $\begin{array}{l}34-55 \\
64-83 \\
59-79 \\
56-81\end{array}$ & $\begin{array}{l}\text { Cuarto de } \\
\text { familiares } \\
\text { Sala de } \\
\text { emergencias } \\
\text { Salas de cirugía } \\
\text { general } \\
\text { Hospitalización }\end{array}$ \\
\hline $\mathrm{Pai}^{24}$ & 2007 & Taiwán & 1,650 & $50-68$ & $\begin{array}{l}\text { Hospitalización } \\
\text { y estaciones de } \\
\text { trabajo }\end{array}$ \\
\hline Otenio et $\mathrm{al}^{25}$ & 2007 & Brasil & 222 & $\begin{array}{l}64 \\
61 \\
58-65 \\
59 \\
58-66\end{array}$ & $\begin{array}{l}\text { Sala de } \\
\text { emergencias } \\
\text { Sala de yesos } \\
\text { ortopédicos } \\
\text { Sala de } \\
\text { cuidados } \\
\text { intensivos } \\
\text { Sala de cirugía } \\
\text { Farmacia }\end{array}$ \\
\hline Mendoza-Sánchez et al ${ }^{26}$ & 1996 & México & 800 & $50-59$ & Hospitalización \\
\hline
\end{tabular}

Tabla 2 Promedios de ruido ambiental, valores mínimos y máximos por servicio y turno

\begin{tabular}{llllll}
\hline Servicio & Turno & Ruido $(\mathrm{dB})$ Promedio $\pm \mathrm{DE}$ & Mínimo & Máximo & $\mathrm{p}^{*}$ \\
\hline UCIC & A & $60.20 \pm 1.58$ & 58.38 & 61.17 & 0.275 \\
& B & $59.25 \pm 1.99$ & 57.03 & 60.89 & 0.513 \\
$\mathrm{UCI}$ & $\mathrm{A}$ & $64.77 \pm 3.33$ & 60.97 & 67.18 & 64.34 \\
& $\mathrm{~B}$ & $62.83 \pm 1.62$ & 61.10 & \\
\hline
\end{tabular}

A: entre semana; B: fin de semana; dB: decibelios; DE: desviación estándar; UCI: unidad de cuidados intensivos; UCIC: unidad de cuidados intensivos coronarios.

" Valor de p calculado mediante prueba U de Mann-Whitney.

Tabla 3 Valores de ruido que sobrepasan el nivel máximo permitido por la Organización Mundial de la Salud (45dB) en los servicios evaluados

\begin{tabular}{lllllllll}
\hline Servicio & Turno & Ruido $(\mathrm{dB}) \mu \pm \mathrm{DE}$ & P5 & P10 & P25 & P50 & P75 & $\mathrm{P}^{*}$ \\
\hline UCIC & A & $60.20 \pm 1.58$ & 58.38 & 58.38 & 59.72 & 61.07 & 61.12 & 0.275 \\
& B & $59.25 \pm 1.99$ & 57.03 & 57.03 & 58.43 & 59.83 & 60.36 \\
UCI & A & $64.77 \pm 3.33$ & 60.97 & 60.97 & 63.57 & 66.18 & 66.68 \\
& B & $62.83 \pm 1.62$ & 61.10 & 61.10 & 62.06 & 63.03 & 63.68 \\
\hline
\end{tabular}

A: entre semana; B: fin de semana; dB: decibelios; DE: desviación estándar; P5, P10, P25, P50, P75: percentiles 5, 10, 25, 50, 75; UCI: unidad de cuidados intensivos; UCIC: unidad de cuidados intensivos coronarios; $\mu \pm \mathrm{DE}$ : promedio \pm desviación estándar.

"Valor de p calculado mediante prueba de Kruskal-Wallis.

dentro del hospital; es posible que sea un factor contribuyente que incrementa la morbilidad en los pacientes ${ }^{28}$.

Usualmente, los niveles de ruido en un hospital que se considera tranquilo pueden ir de 40 a $50 \mathrm{~dB}$, en un hospital moderadamente ruidoso de 50 a $60 \mathrm{~dB}$ y en un centro hospitalario muy ruidoso de $60 \mathrm{a} 70 \mathrm{~dB}$. Por lo tanto, con los resultados obtenidos nuestro hospital se ubica en el rango de un hospital muy ruidoso. Este dato es consistente con 
lo reportado por otros estudios similares en hospitales del mundo $20,29-33$.

La conversación entre el personal de las unidades de cuidados críticos puede generar niveles de ruido iguales 0 mayores a $52 \mathrm{~dB}$ en el $55 \%$ de los casos de ruido excesivo ${ }^{34}$. En este estudio y de forma informal se indagó entre el personal de enfermería acerca de su opinión del efecto que produce el platicar durante su trabajo; la respuesta en la mayoría del personal fue que no consideraban que eso tuviera algún efecto en la atención de los enfermos. A este respecto, es posible afirmar que una unidad de cuidados críticos excesivamente ruidosa donde el personal no se preocupa por mantener niveles bajos de ruido no es el mejor escenario para tratar a un enfermo; y así lo concluyeron GómezCarretero et al. ${ }^{35}$, en un estudio realizado en 2007, al revisar el efecto de las alarmas, los equipos de aspiración, los teléfonos, y las conversaciones del equipo de la $\mathrm{UCl}$ sobre el sueño; encontraron que las pláticas entre el personal eran una fuente de ruido y confusión ${ }^{36}$, ya que al no poder valorar adecuadamente el nivel de conciencia del enfermo es posible que en su estado puedan llegar a entender parcialmente las conversaciones, que en algunas ocasiones pueden estresarles, generando miedo y ansiedad ${ }^{33}$.

Al comparar el nivel de ruido encontrado en las UCC de nuestro centro, se pudo establecer que es comparable al nivel de ruido observado en una oficina bulliciosa $(60-70 \mathrm{~dB})^{25,37,38}$. Esto representa $25-30 \mathrm{~dB}$ por encima de lo permitido y que en gran medida puede ser el resultado de un incremento en los niveles de conversación entre familiares, personal del hospital y ruido de equipos médicos. Es necesario mantener acciones permanentes entre familiares y personal del hospital para asegurar la disminución de contaminación por ruido originada por este motivo. En nuestro hospital los niveles de ruido fueron siempre mayores en la $\mathrm{UCl}$, situación que puede explicarse por un mayor flujo de pacientes, personal y familiares dentro de esa área. La causa indudable de este hecho fue que existe un menor número de pacientes hospitalizados en la $\mathrm{UCIC}$ con respecto de la $\mathrm{UCI}$ que cuenta con el doble de número de camas disponibles.

Este estudio presenta las siguientes limitaciones. Primero, se recolectaron lecturas de ruido solo en 3 momentos diferentes que correspondían a los tiempos de mayor trabajo y flujo de personas dentro de las áreas estudiadas, no se tomaron en cuenta las variaciones más bajas que pudieran haber ocurrido en cada uno de las áreas estudiadas. En segundo lugar, el medidor utilizado discriminaba dentro de un rango de 40-130 dB; sin embargo, no fue posible examinar aquellos ruidos con una intensidad menor a $40 \mathrm{~dB}$, aunque la finalidad de este estudio estaba enfocada más en explorar los niveles de ruido más intensos que se han asociado con problemas de salud y que afectan la calidad de la atención dentro de los hospitales. En tercer lugar, el número de pacientes hospitalizados varía en cada una de las áreas estudiadas y quizá esto influyó en un mayor nivel de ruido en la $\mathrm{UCI}$ con respecto de la UCIC, ya que se incrementa el número de personal, equipos y personas que circulan en áreas con un mayor número de pacientes. Cuarto, no recolectamos información acerca de factores fisiológicos que pudieran ser afectados en los enfermos, como la calidad sueño, presión arterial y grado de estrés entre otros. Quinto, no evaluamos las diferencias acerca del nivel de actividad alrededor de la cama del enfermo de forma individual, ni el contenido espectral del ruido ambiental. Esta información podría ser muy útil para conocer aquellos dispositivos o situaciones que incrementan más el ruido ambiental y podría ser una excelente propuesta para estudios posteriores en este tema.

Se ha descrito que hasta un $51 \%$ del ruido que ocurre en una UCC es modificable ${ }^{18}$. En un estudio realizado por Tegnestedt et al. en 2013, se concluye que es durante la noche cuando existen los niveles de ruido más bajos, y su origen está asociado más al ruido producido por alarmas y conversaciones entre el personal sanitario, y no a los cuidados del paciente ${ }^{39}$. Por lo tanto, dentro de las acciones que sugerimos para la disminución del ruido se encuentran: jornadas de educación al personal sobre los efectos del ruido en los enfermos, disponer de carteles alusivos a la importancia de mantener niveles bajos de ruido dentro del hospital, limitar las conversaciones cerca de los enfermos, reducir la intensidad de las alarmas en monitores ${ }^{30}$, disminuir la intensidad de la luz ${ }^{40}$, responder rápida y oportunamente a las alarmas sonoras, instruir al personal para que responda el teléfono de forma inmediata y atenuar la intensidad del timbre, no cerrar puertas y ventanas de forma violenta ${ }^{18} \mathrm{y}$ colocar soportes para reducir los golpes, evitar el choque de mesas, sillas y soportes de soluciones contra la cama del paciente, modificar rutas de limpieza, paso de carritos repartidores de ropa y comida, no utilizar la radio ${ }^{35}$, televisión ${ }^{35}$ o teléfonos celulares dentro de la UCC, limitar o reducir las visitas de familiares y amigos, así como ubicar a los enfermos más graves lejos de las áreas más congestionadas o ruidosas. Las sugerencias expresadas son de fácil aplicación y no implican mayores costos para el hospital, sin embargo, podrán generar un cambio trascendental en el ambiente intrahospitalario para todos.

\section{Conclusiones}

Este sencillo estudio concluye que existen niveles elevados de ruido tanto en la UCIC como en la UCI de nuestro centro hospitalario y que superan lo recomendado por la Organización Mundial de la Salud. Es un problema poco estudiado en México e Iberoamérica. Es urgente realizar acciones encaminadas a lograr una mayor participación del personal en la disminución del ruido, así como la realización de estudios que permitan analizar el efecto individual de las alarmas sonoras sobre el enfermo, la posición de los equipos de monitoreo para la generación de ruido y aquellas intervenciones sobre el enfermo que incrementan el ruido, así como los posibles efectos en la salud.

\section{Responsabilidades éticas}

Protección de personas y animales. Los autores declaran que los procedimientos seguidos se conformaron a las normas éticas del comité de experimentación humana responsable y de acuerdo con la Asociación Médica Mundial y la Declaración de Helsinki.

Confidencialidad de los datos. Los autores declaran que han seguido los protocolos de su centro de trabajo sobre la publicación de datos de pacientes. 
Derecho a la privacidad y consentimiento informado. Los autores han obtenido el consentimiento informado de los pacientes y/o sujetos referidos en el artículo. Este documento obra en poder del autor de correspondencia.

\section{Financiación}

La fuente de financiación fue con recursos propios. No se tuvo intervención de ninguna empresa o fuente externa al Instituto Mexicano del Seguro Social.

\section{Conflicto de intereses}

Los autores declaran que no existió conflicto de intereses en la realización de este estudio.

\section{Agradecimientos}

Al Instituto Mexicano del Seguro Social por permitirnos realizar este estudio dentro de las áreas intrahospitalarias. Muy en especial, los autores quieren agradecer el apoyo técnico en seguridad e higiene de la ingeniera Josefina Verdugo Urías en la lectura e interpretación de las mediciones de ruido.

\section{Bibliografía}

1. Pérez-Cárdenas MD, Rodríguez-Gómez M, Fernández-Herranz Al, et al. Valoración del grado de satisfacción de los familiares de pacientes ingresados en una unidad de cuidados intensivos. Med Intensiva. 2004;28:237-49.

2. Schmid F, Goepfert MS, Reuter DA. Patient monitoring alarms in the ICU and in the operating room. Crit Care. 2013;17:216.

3. World Health Organization. WHO Guidelines for Community Noise. 1999.

4. Marqués P, Calvo D, Mompart MP, et al. Multi-center study of noise in patients from hospitals in Spain: A questionnaire survey. Noise Health. 2012;14:83-5.

5. Pereira RP, Toledo RN, Amaral JLG, et al. Qualification and quantification of ambient noise exposure in a general intensive care unit. Rev Bras Otorrinolaringol. 2003;69:766-71.

6. Freedman NS, Kotzer N, Schwab RJ. Patient perception of sleep quality and etiology of sleep disruption in the intensive care unit. Am J Respir Crit Care Med. 1999;159:1155-62.

7. Brandenberger G, Follenius M, Wittersheim G, et al. Plasma catecholamines and pituitary adrenal hormones related to mental task demand under quiet and noise conditions. Biol Psychol. 1980;10:239-52.

8. Stansfeld SA, Matheson MP. Noise pollution: Non-auditory effects on health. Br Med Bull. 2003;68:243-57.

9. Kaltenbach M, Maschke C, Klinke R. Health consequences of aircraft noise. Dtsch Arztebl Int. 2008;105:548-56.

10. Hume KI, Brink M, Basner M. Effects of environmental noise on sleep. Noise Health. 2012;14:297-302.

11. Melamed S, Froom P, Kristal-Boneh E, et al. Industrial noise exposure, noise annoyance, and serum lipid levels in blue-collar workers-the CORDIS Study. Arch Environ Health. 1997;52:292-8.

12. Babisch W, Gallacher JEJ, Elwood PC, et al. Traffic noise and cardiovascular risk. The Caerphilly Study, first phase: Outdoor noise levels and risk factors. Arch Environ Health. 1988;43:407-14.

13. Lawson N, Thompson K, Saunders G, et al. Sound intensity and noise evaluation in a critical care unit. Am J Crit Care. 2010;19:e88-99.
14. Aaron JN, Carlisle CC, Carskadon MA, et al. Environmental noise as a cause of sleep disruption in an intermediate respiratory care unit. Sleep. 1996;19:707-10.

15. Scott A. Managing anxiety in ICU patients: The role of preoperative information provision. Nurs Crit Care. 2004;9:72-9.

16. Nicolás A, Aizpitarte E, Iruarrizaga A, et al. Perception of nighttime sleep by the surgical patients in an Intensive Care Unit. Nurs Crit Care. 2008;13:25-33.

17. Richards KC, O'Sullivan PS, Phillips RL. Measurement of sleep in critically ill patients. J Nurs Meas. 2000;8:131-44.

18. Kahn DM, Cook TE, Carlisle CC, et al. Identification and modification of environmental noise in an ICU setting. Chest. 1998;114:535-40.

19. Busch-Vishniac IJ, West JE, Barnhill C, et al. Noise levels in Johns Hopkins Hospital. J Acoust Soc Am. 2005;118:3629-45.

20. Vinodhkumaradithyaa A, Srinivasan M, Ananthalakshmi I, et al. Noise levels in a tertiary care hospital. Noise Health. 2008;10:11-3.

21. Vílchez-Dagostino P, Porras-Peña K, Giles-Saavedra R, et al. Correlation of anxiety and acoustic contamination in hospitalized patients of the hospital Almanzor Aguinaga Asenjo, june 2010. Rev cuerpo méd. HNAAA. 2012;5:10-5.

22. Yoder JC, Staisiunas PG, Meltzer DO, et al. Noise and sleep among adult medical inpatients: Far from a quiet night. Arch Intern Med. 2012;172:68-70.

23. McLaren E, Maxwell-Armstrong C. Noise pollution on an acute surgical ward. Ann R Coll Surg Engl. 2008;90:136-9.

24. Pai JY. A study in hospital noise-a case from Taiwan. Int J Occup Saf Ergon. 2007;13:83-90.

25. Otenio MH, Cremer E, Claro EM. Noise level in a 222 bed hospital in the 18th health region-PR. Braz J Otorhinolaryngol. 2007;73:245-50.

26. Mendoza-Sánchez RS, Roque-Sánchez RH, Moncada-González B. Noise level in a care and teaching hospital institution. Gac Med Mex. 1996;132:127-33.

27. Konkani A, Oakley B. Noise in hospital intensive care units-a critical review of a critical topic. J Crit Care. 2012;27:522, e1-9.

28. Macedo IS, Mateus DC, Costa Ede M, et al. Noise assessment in intensive care units. Braz J Otorhinolaryngol. 2009;75:844-6.

29. Park MJ, Yoo JH, Cho BW, et al. Noise in hospital rooms and sleep disturbance in hospitalized medical patients. Environ Health Toxicol. 2014;29:e2014006.

30. Darbyshire JL, Young JD. An investigation of sound levels on intensive care units with reference to the WHO guidelines. Critical Care. 2013;17:R187.

31. Xie H, Kang J. The acoustic environment of intensive care wards based on long period nocturnal measurements. Noise Health. 2012;14:230-6.

32. Khademi G, Roudi M, Shah Farhat A, et al. Noise pollution in intensive care units and emergency wards. Iran J Otorhinolaryngol. 2011;23:141-8.

33. Elliott RM, McKinley SM, Eager D. A pilot study of sound levels in an Australian adult general intensive care unit. Noise Health. 2010;12:26-36

34. Bentley S, Murphy F, Dudley H. Perceived noise in a surgical Ward and an intensive care unit: An objective analysis. Br Med J. 1977;2:1503-6.

35. Gómez-Carretero P, Monsalve V, Soriano JF, et al. Emotional disorders and psychological needs of patients in an Intensive Care Unit. Med Intensiva. 2007;31:318-25.

36. Ryherd EE, Waye KP, Ljungkvist L. Characterizing noise and perceived work environment in a neurological intensive care unit. J Acoust Soc Am. 2008;123:747-56.

37. Xie H, Kang J, Mills GH. Clinical review: The impact of noise on patients' sleep and the effectiveness of noise reduction strategies in intensive care units. Crit Care. 2009;13:208.

38. Richardson A, Thompson A, Coghill E, et al. Development and implementation of a noise reduction intervention programme: 
A pre- and postaudit of three hospital wards. J Clin Nurs. 2009; $18: 3316-24$.

39. Tegnestedt C, Günther A, Reichard A, et al. Levels and sources of sound in the intensive care unit - an observational study of three room types. Acta Anaesthesiol Scand. 2013;57: 1041-50.

40. Novaes MA, Aronovich A, Ferraz MB, et al. Stressors in ICU: Patients' evaluation. Intensive Care Med. 1997;23:1282-5. 